\title{
Perspectives and diagnostic considerations in spinal muscular atrophy
}

\author{
Thomas W. Prior, PhD
}

TABLE OF CONTENTS

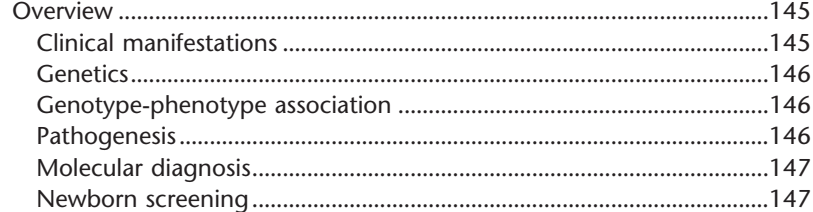

\begin{abstract}
Spinal muscular atrophy is an autosomal recessive neurodegenerative disease and the most common genetic cause of infant mortality. The disease results in motor neuron loss and skeletal muscle atrophy. Spinal muscular atrophy is caused by mutations in the telomeric copy of the survival motor neuron 1 (SMN1) gene, but all patients retain a centromeric copy of the gene, $S M N 2$. In the majority of cases, the disease severity correlates inversely with an increased SMN2 gene copy number. Because spinal muscular atrophy is both a severe and common disorder, a direct carrier testing has been beneficial to many families. The survival motor neuron protein is ubiquitously expressed and performs a role in the assembly of the spliceosome. It is still not understood why mutations in the SMN1 gene only seem to affect motor neurons. Progress has been made by developing therapeutic strategies based on understanding the pathogenesis of the disease. This review attempts to highlight some of the recent advances in the understanding of the disease with a focus on molecular diagnostics. Genet Med 2010: 12(3):145-152.
\end{abstract}

Key Words: spinal muscular atrophy, survival motor neuron, SMN1, SMN2, genetic testing, carrier testing

\section{OVERVIEW}

Spinal muscular atrophy (SMA) is an autosomal recessive neuromuscular disorder caused by mutations in the survival motor neuron (SMN1) gene. SMA is the second most common fatal autosomal recessive disorder after cystic fibrosis (CF), with an estimated incidence of $\sim 1$ in 10,000 live births. ${ }^{1}$ The disease is characterized by progressive symmetrical muscle weakness resulting from the degeneration and loss of anterior horn cells in the spinal cord and brain stem nuclei. The disease is classified on the basis of age of onset and clinical course. Two almost identical SMN genes are present on 5q13: the SMN1

From the Department of Pathology, The Ohio State University, Columbus, Ohio

Thomas W. Prior, PhD, Department of Pathology, The Ohio State University, 125 Hamilton Hall, 1645 Neil Avenue, Columbus, OH 43210. E-mail: thomas.prior@osumc.edu.

Disclosure: The author declares no conflict of interest.

Submitted for publication August 17, 2009.

Accepted for publication October 11, 2009.

Published online ahead of print January 6, 2010.

DOI: 10.1097/GIM.0b013e3181c5e713

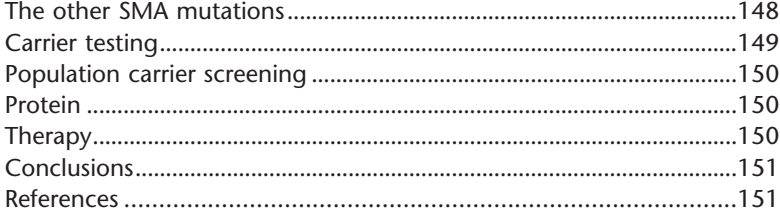

gene, which is the SMA-determining gene, and the SMN2 gene. The homozygous absence of the SMN1 exon 7 has been observed on the majority of patients, whereas the remaining patients have intragenic SMN1 mutations. Although SMN2 produces less full-length transcript than SMN1, the number of SMN2 copies modulates the phenotype. Carrier detection, in the deletion cases, relies on the accurate determination of the SMN1 gene copies. Because SMA is one of the most common lethal genetic disorders, with a carrier frequency of $1 / 40-1 / 60$, a direct carrier dosage testing has been beneficial to many families. The American College of Medical Genetics has recently recommended population carrier screening for SMA. ${ }^{2}$ The management of SMA involves supportive and preventive strategies. New treatments based on increasing the expression of full-length SMN protein levels from the SMN2 gene are being investigated.

\section{Clinical manifestations}

The autosomal recessive disorder proximal SMA (Types I, II, and III [OMIM 253300, 253550, and 253400]) is a severe neuromuscular disease characterized by degeneration of alpha motor neurons in the spinal cord, which results in progressive muscle weakness and paralysis. The predominant pathologic feature of autopsy studies of patients with SMA is loss of motor neurons in the ventral horn of the spinal cord and in brain stem motor nuclei. SMA is the second most common fatal autosomal recessive disorder after $\mathrm{CF}$, with an estimated incidence of $\sim 1$ in 10,000 live births. ${ }^{1}$ Childhood SMA is subdivided into three clinical groups on the basis of age of onset and clinical course. . $^{3,4}$ Type I SMA (Werdnig-Hoffman disease) is characterized by severe, generalized muscle weakness, and hypotonia at birth or within the first 3 months. Death from respiratory failure usually occurs within the first 2 years. Approximately $60 \%$ to $70 \%$ of patients with SMA have the type I disease. ${ }^{5}$ Type II children are able to sit, although they cannot stand or walk unaided, and typically survive beyond 4 years. The phenotypic variability exceeds that observed in type I patients, ranging from infants who sit transiently and demonstrate severe respiratory insufficiency to children who can sit, crawl, and even stand with support. Prognosis in this group is largely dependent on the degree of respiratory involvement. Type III SMA (Kugelberg-Welander disease) is a milder form, with onset during infancy or youth: patients learn to walk unaided and have prolonged survivals. They comprise a less fragile group than type II patients with regard to respiratory and nutritional vulnerability. Type III SMA is further subdivided into 
two groups, type IIIa (onset before 3 years) and type IIIb (onset at age $\geq 3$ years). Cases presenting with the first symptoms of the disease at the age of 20 to 30 years are classified as type IV or proximal adult-type SMA. The described classification is based on age of onset and clinical course, but it should be recognized that the disorder demonstrates a continuous range of severity. Finally, although the disease affects both sexes equally, there have been reports that the severe type I is more common in female subjects and that female subjects are less affected than male subjects in the milder SMA types. ${ }^{6,7}$

\section{Genetics}

Linkage analysis mapped all clinical types of SMA to chromosome 5q11.2-13.3.8,9 The SMA gene is within a complex region, containing multiple repetitive and inverted sequences. ${ }^{10}$ The $S M N$ gene (Entrez Gene ID number 6606) comprises nine exons with a stop codon present near the end of exon 7.11 Two inverted $S M N$ copies are present: the telomeric or $S M N 1$ gene, which is the SMA-determining gene, and the centromeric or $S M N 2$ gene. The two SMN genes are highly homologous, have equivalent promoters, ${ }^{12,13}$ and only differ at five base pairs. ${ }^{10}$ The base differences are used to differentiate SMN1 from SMN2. The coding sequence of SMN2 differs from that of SMN1 by a single nucleotide $(840 \mathrm{C}>\mathrm{T})$, which does not alter the amino acid sequence but has been shown to be important in splicing. Both copies of the SMN1 exon 7 are absent in $\sim 95 \%$ of affected patients. Although patients with SMA lack SMN1, they always carry at least one copy of $S M N 2$, which is partially functional but unable to compensate for the absence of SMN1. The remaining $5 \%$ of affected cases are compound heterozygotes for SMN1 exon 7 deletion and small intragenic mutations.

\section{Genotype-phenotype association}

Although mutations of the SMN1 gene are observed in the majority of patients, no genotype-phenotype correlation was initially observed because SMN1 exon 7 is absent in the majority of patients independent of the severity of SMA. This is due to the fact that routine diagnostic methods do not distinguish between a deletion of $S M N 1$ and a conversion event whereby $S M N 1$ is replaced by a copy of SMN2. Several studies have now shown that the SMN2 copy number modifies the severity of the disease. ${ }^{14-17}$ The copy number varies from zero to three copies in the normal population, with $\sim 10 \%$ to $15 \%$ of controls having no SMN2. However, milder patients with type II or III SMA have been shown to have more copies of SMN2 than type I patients. A majority of patients with the severe type I form have one or two copies of SMN2, most patients with type II have three $S M N 2$ copies, and most patients with type III have three or four SMN2 copies. Three unaffected family members of patients with SMA, with confirmed SMN1 homozygous deletions, were shown to have five copies of SMN2. ${ }^{18}$ These cases not only support the role of SMN2 modifying the phenotype but also demonstrate that expression levels consistent with five copies of the SMN2 genes may be enough to compensate for the absence of the SMN1 gene.

This inverse dose relationship between SMN2 copy number and disease severity has also been supported by the SMA mouse model. ${ }^{19,20}$ The SMA mouse models have not only confirmed the susceptibility of motor neuron degeneration to $S M N$ deficiency but also verified that the degeneration can be prevented by increased $S M N 2$ dosage. Mice lacking the endogenous mouse Smn gene, but expressing two copies of the human SMN2 gene develop severe SMA and die within 1 week of age, however, mice that express multiple copies of SMN2 do not develop the disease.
In addition to the SMN2 copy number, other modifying factors influence the phenotypic variability of SMA. There are very rare families reported in which markedly different degrees of disease severity are present in affected siblings with the same SMN2 copy number. These discordant sib pairs, which share the same genetic background around the SMA locus, would indicate that there are other modifier genes outside the SMA region. Differences in splicing factors may allow more full-length expression from the SMN2 gene and account for some of the variability observed between discordant sibs. ${ }^{21}$ It was also found that in some rare families with unaffected SMN1-deleted females, the expression of plastin 3 (PLS3) was higher than in their SMA-affected counterparts. ${ }^{22} P L S 3$ was shown to be important for axonogenesis and therefore may act as a protective modifier. However, the exact role of PLS3 in SMA needs further confirmation and PLS3 will need to be expressed in the SMA mouse model to determine whether the lethality can be corrected.

Still, it remains unclear whether these are always fully intact copies of the SMN2 gene in these patients. Because of the nature of the $S M N$ loci, deletion break points have been difficult to identify. If the $S M N 2$ genes are truncated, they may not produce full-length transcripts and therefore be nonfunctional. The identification of gene modifiers not only provides important insight into pathogenesis of SMA but may also identify potential targets for therapy. Several drugs have been shown to increase SMN2 expression in SMA patient-derived cell lines and are being studied in ongoing clinical trials (described "Therapy" section).

\section{Pathogenesis}

Because all individuals with SMA have at least one SMN2 gene copy and there are no differences in the amino acid sequence between the two genes, the obvious question that arises is why do individuals with SMN1 mutations have a SMA phenotype? It has been shown that the SMN1 gene produces full-length transcript, whereas the SMN2 gene produces predominantly an alternatively spliced transcript (exon 7 deleted) encoding a protein (SMN $\Delta 7$ ) that does not oligomerize efficiently and is unstable. ${ }^{23,24}$ The inclusion of exon 7 in SMN1 transcripts and exclusion of this exon in SMN2 transcripts are caused by the single nucleotide difference at +6 in $S M N 1$ exon $7(\mathrm{c} .840 \mathrm{C}>\mathrm{T})$. Although the $\mathrm{C}$ to $\mathrm{T}$ change in $S M N 2$ exon 7 does not change an amino acid, it does disrupt an exonic splicing enhancer (ESE) or creates an exon silencer element (ESS) that results in the majority of transcripts lacking exon 7. ${ }^{25,26}$ The ESEs and ESSs are cis-acting exonic sequences that influence the use of flanking splice sites. ESEs stimulate splicing and are often required for efficient intron removal, whereas ESSs inhibit splicing. Whether it will be the loss of an ESE or creation of an ESS, the result is a reduction of full-length transcripts generated from $S M N 2$. A single SMN2 gene produces less functional protein compared with a single SMN1 gene. ${ }^{27-29}$ Therefore, SMA arises because the SMN2 gene cannot fully compensate for the lack of functional SMN when SMN1 is mutated. However, small amounts of full-length transcripts generated by SMN2 are able to produce a milder type II or III phenotype when the copy number of the SMN2 gene is increased (Fig. 1). SMA is, therefore, caused by low levels of SMN protein, rather than a complete absence of the protein.

Finally, a recent report described three unrelated patients with SMA who possessed SMN2 copy numbers that did not correlate with the observed mild clinical phenotypes. ${ }^{30}$ A single base substitution in $S M N 2$, c.859G $>$ C, was identified in exon 7 in the patients DNA, and it was shown that the substitution 


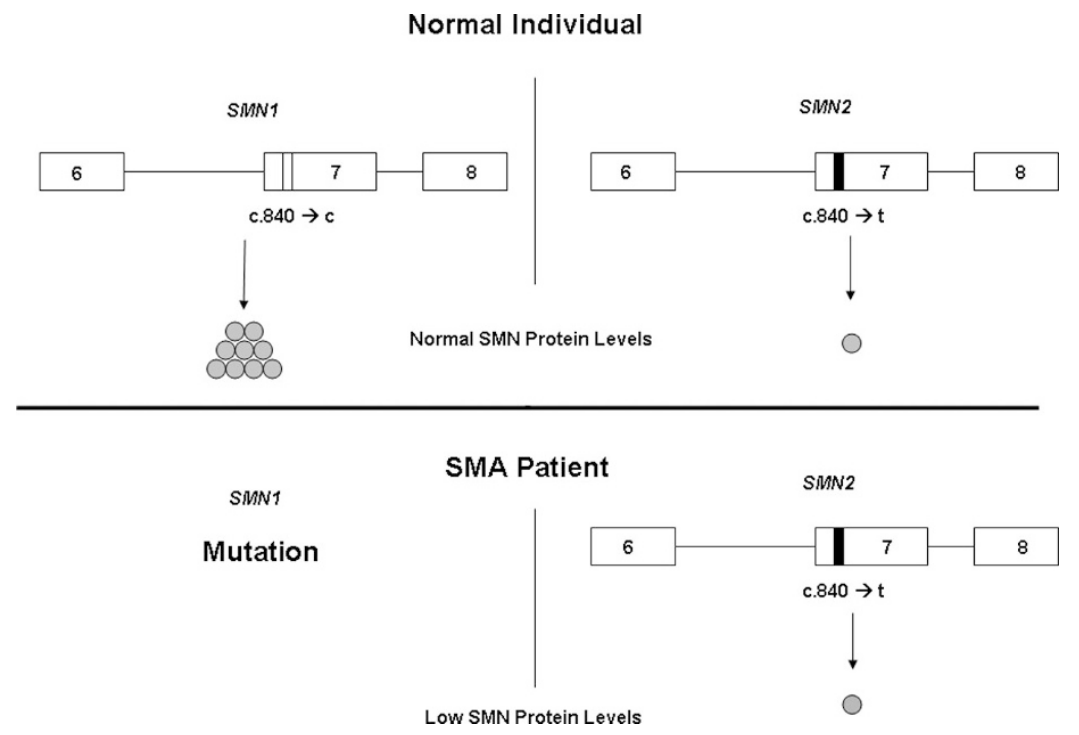

Fig. 1. In normal individuals, most full-length SMN transcript and protein are generated from the SMN1 gene. Patients with SMA have homozygous mutations of SMN1 but retain at least one copy of the SMN2 gene. During transcription of $S M N 2$, the SMN2 gene produces predominantly an alternative transcript (exon 7 deleted) encoding a protein (SMN $\Delta 7$ ) that does not oligomerize efficiently and is unstable. Small amounts of full-length transcripts generated by SMN2 are able to produce a milder Type II or III phenotype when the copy number of the SMN2 gene is increased. SMA results from low levels of SMN protein, rather than a complete absence of the protein.

created a new ESE element. The new ESE increased the amount of exon 7 inclusion and full-length transcripts generated from $S M N 2$, thus resulting in the less severe phenotypes. Therefore, the SMA phenotype may not only be modified by the number of $S M N 2$ genes but SMN2 sequence variations can also affect the disease severity. It should, therefore, not be assumed that all $S M N 2$ genes are equivalent and sequence changes found within the SMN2 gene must be further investigated for potential positive or negative effects on $S M N 2$ transcription.

\section{Molecular diagnosis}

The first diagnostic test for a patient suspected to have SMA should be the $S M N$ gene deletion test. Both copies of the SMN1 exon 7 are absent in $\sim 95 \%$ of affected patients, whereas small more subtle mutations have been identified in the remaining affected patients. The molecular diagnosis of SMA consists of the detection of the absence of exon 7 of the SMN1 gene. Although this is a highly repetitive region with the almost identical $S M N 2$ copy, the exon 7 base pair difference $(840 \mathrm{C}>\mathrm{T}$ ) alters a restriction enzyme site (because of a mismatched primer) and allows one to easily distinguish $S M N 1$ from the $S M N 2$ using a polymerase chain reaction (PCR)-based assay (Fig. 2). As shown in Figure 2, the restriction enzyme DraI cuts only the $S M N 2$ exon 7 PCR products, and in patients with SMA, the uncut SMNI exon 7 is absent. ${ }^{31}$ The absence of detectable SMN1 in patients with SMA is being used as a reliable and powerful diagnostic test for the majority of patients with SMA. SMN2 is homozygously absent in $\sim 5 \%$ to $10 \%$ of unaffected individuals. ${ }^{10,15}$ The SMA deletion test is currently being performed by several diagnostic laboratories and the result can easily be obtained in 2 weeks. Limitations of the diagnostic test are the failure to detect the nondeletion mutations of SMNI gene and the inability to determine carrier status.

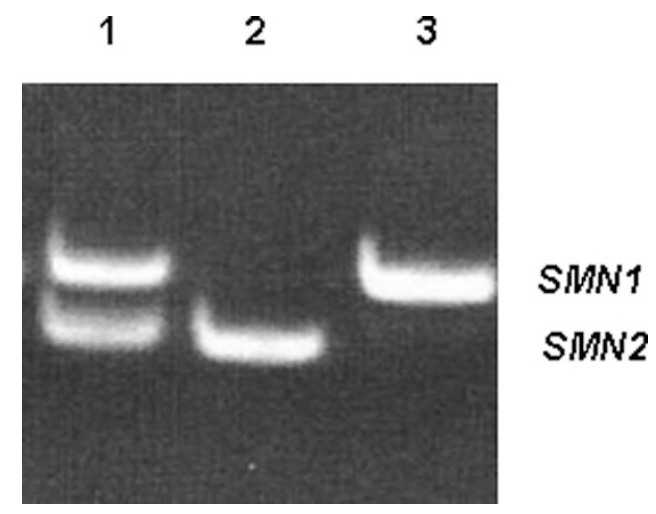

Fig. 2. Restriction enzyme digestion of PCR products distinguishes SMN1 from SMN2 exon 7. Lanes 1: unaffected patients; Lane 2: patient with SMA with SMN1 deletion; and Lane 3: unaffected patient with SMN2 deletion.

\section{Newborn screening}

Although a number of potential therapies are currently in clinical trials (see "Therapy" section), their success may depend on identifying individuals as early as possible to begin treatment before potentially irreversible neuronal loss. In infants with type I SMA, rapid loss of motor units occurs in the first 3 months and severe denervation with loss or $>95 \%$ of units within 6 months. ${ }^{32}$ Therefore, a very small window for beneficial therapeutic intervention exists in infants with type I SMA, and therapies will need to be administered within the newborn period for maximum benefit. This could potentially be accomplished through a newborn screening program for SMA. The identification of patients with SMA during the newborn period 


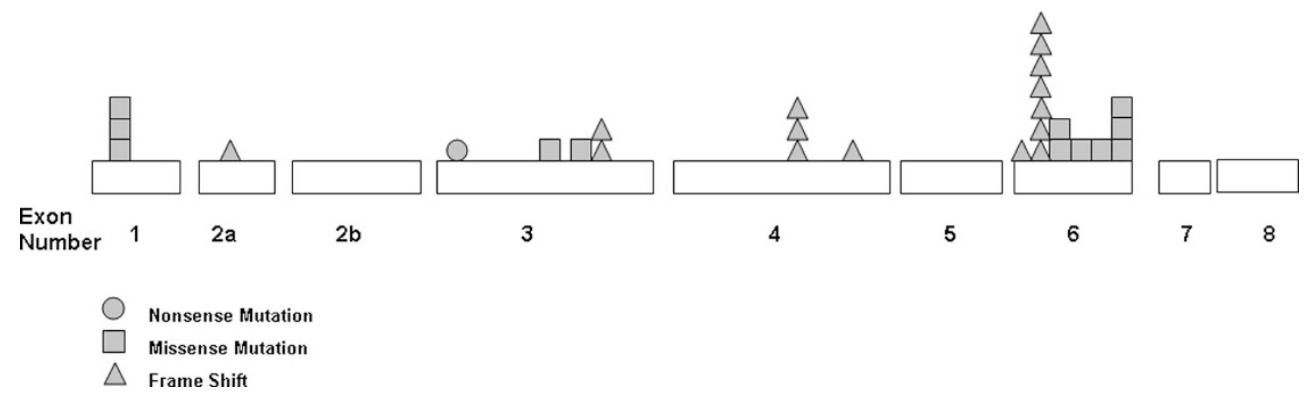

Fig. 3. Distribution of intragenic small mutations in the SMN1 gene. The SMN1 gene has nine exons and there is a hot spot for mutations in Exon 6. Table 1 specifies the actual nucleotide changes.

can be accomplished only by DNA testing because the disorder does not have a biochemical marker. Thus, SMA presents a unique challenge because the testing requires DNA as the substrate, which differs from current practices. However, direct DNA testing is the next innovation in newborn screening but currently is used primarily for reflex testing for first-tier-positive results. With its sizeable capacity for multiplexing, array technology has been touted as the application of choice for the first-tier analysis of DNA in newborn screening. ${ }^{33,34}$ Using liquid microbead array for the detection of the homozygous SMN1 exon 7 deletion, Pyatt et al. ${ }^{35}$ demonstrated that newborn screening for SMA can be technically accomplished. In a series of blood spots, all 164 affected samples were correctly found to have the homozygous SMN1 deletion, whereas 157 unaffected samples were excluded. ${ }^{35}$

There is a growing consensus that newborn screening can be extremely valuable for genetic conditions for which we do not have a specific effective treatment. A newborn screening program for SMA would not only allow patients to be enrolled in the clinical trials at the earliest time period but would enable patients to obtain proactive treatment earlier in the disease progression with regard to nutrition, physical therapy, and respiratory care. Furthermore, identifying SMA-afflicted individuals at birth eliminates the pain and cost of unnecessary testing that often takes place in attempting to diagnose an affected patient. The results from newborn screening are also important for the child's family because of the possibility for the prevention of additional cases through genetic counseling and carrier testing of at-risk family members.

\section{The other SMA mutations}

Although the absence of both copies of the SMN1 gene is a very reliable and sensitive assay for the molecular diagnosis of SMA, $\sim 5 \%$ of affected patients have other types of mutations in the SMN1 gene that will not be detected by homozygous deletion testing. Because of the high deletion frequency and according to the Hardy-Weinberg equilibrium, most of these patients will be compound heterozygotes, with one $S M N 1$ allele being deleted and the other allele with a point mutation or other types of small mutations. If a patient with SMA possesses only a single copy of $S M N 1$, it is likely that the remaining copy contains a more subtle mutation, including nonsense mutations, missense mutations, splice site mutation insertions, and small deletions. Figure 3 and Table 1 present the small intragenic mutations identified at The Ohio State University Molecular Pathology Laboratory. These intragenic types of mutations provided strong support that mutations in the SMN1 gene alone can produce the disease. This was important because there was initial speculation that genes in close proximity, which were

\begin{tabular}{lllc}
\hline Table 1 & SMN1 mutations & & \\
\hline Exon & \multicolumn{1}{c}{ Mutation } & Type & No. patients \\
\hline 1 & c.5 C $>$ G; p.A2G & Missense & 3 \\
$2 \mathrm{a}$ & c.109insA & Frameshift & 1 \\
3 & c.305 G $>$ A; p.WIO2X & Nonsense & 1 \\
3 & c.389A $>$ G; p.YI3OC & Missense & 1 \\
3 & c.4I8-432del15 & Frameshift & 2 \\
3 & c.419 A $>$ T; p. DI4OV & Missense & 1 \\
4 & c.509-510delGT & Frameshift & 3 \\
4 & c.584delC & Frameshift & 1 \\
6 & c.735insC & Frameshift & 1 \\
6 & c.770-780dup11 & Frameshift & 7 \\
& c.785G $>$ T; p.S2621 & Missense & 2 \\
6 & c.796flC; p.S266P & Missense & 1 \\
6 & c.818A $>$ G; p.H273R & Missense & 1 \\
6 & c.821C $>$ T; p.T2741 & Missense & 3 \\
\hline & & &
\end{tabular}

often also deleted with the SMN1 gene, might modify the phenotype. These mutations have also been essential in defining important structural and functional domains of the SMN protein. As shown in Figure 3, although the mutations are distributed across the gene, there is a hot spot for missense mutations in Exon 6. Exon 6 corresponds to a domain in the protein, which has been shown to be important in protein oligomerization. ${ }^{36}$ Many of the same intragenic mutations have now been reported in unrelated patients. 7,37,38 The most frequently reported mutations are the p.Tyr272Cys, c.399_402delAGAG, c.770780dup11, and p.Thr274Ile. These four mutations were shown to account for $42 \%$ of cases with small changes in the SMN1 gene. ${ }^{7}$ We have observed the c.770-780dup11 in seven unrelated patients.

The characterization of these intragenic SMN1 mutations has provided additional support regarding the role of SMN2 in modifying the phenotype. ${ }^{38}$ The severe type I SMA phenotype is often the result of frameshift mutations that can be ameliorated by an increase in the SMN2 copy number. As a consequence of the $S M N 1$ gene being relatively small, and given the 
uniform spectrum of mutations, it is a relatively straightforward procedure to sequence the gene and identify mutations in patients who are negative for the diagnostic deletion test. However, it is necessary to verify that the intragenic mutation has occurred in the SMN1 gene and not in the SMN2 gene. This requires either a long-range PCR protocol or a subcloning. Finally, the carrier test can be used as an initial and sensitive screen to identify patients heterozygous for the deletion of SMN1 who are likely to also have these smaller types of mutations. If the patient possesses two copies of SMN1, then other motor neuron disorders should be considered such as: SMA with respiratory distress, X-linked SMA, distal muscular atrophy, and juvenile amyotrophic lateral sclerosis.

\section{Carrier testing}

Because SMA is one of the most common lethal genetic disorders, with a carrier frequency of $1 / 40-1 / 60$, a direct carrier testing has been beneficial to many families. Carrier detection for the heterozygous state was initially shown to be more technically challenging because the SMA region is characterized by the presence of many repeated elements. It has been observed that the SMN2 copy number fluctuates: $\sim 10 \%$ to $15 \%$ of controls lack the SMN2 copy, whereas many of the more mildly affected patients with SMA have more copies of it. Thus, a straightforward dosage assay using the SMN2 gene as the internal control would not be reliable. McAndrew et al. ${ }^{15}$ developed the first highly accurate competitive dosage assays for determination of SMA carrier status. In the competitive PCR method, a known number of copies of a synthetic mutated internal standard are introduced with the patient sample into the PCR mixture. The internal standards are designed to be amplified with the same primer pairs for the SMN1 copy, with efficiencies similar to those of the genomic DNA counterparts, and yield PCR products slightly smaller than the SMN PCR product. The copy number of SMN1 is determined by coampli- fication of $S M N 1, S M N 2$, the $S M N$ internal standard, CF, and the CF internal standard and quantitated on an automated sequencer (Fig. 4). The major advantage of this technique is that the internal standard is amplified with the same primers that amplify the target sequence. Thus, the efficiency of the amplification of the patient DNA and the internal standard DNA should be very similar and allow one to accurately determine the gene copy number. The assay can also be used for the identification of the $5 \%$ of SMA-affected individuals who are compound heterozygotes. There have now been a number of techniques developed for the detection of SMA carriers including: real-time PCR, ${ }^{6}$ competitive PCR with primer extension, ${ }^{39}$ TaqMan technology, ${ }^{40}$ denaturing high-performance liquid chromatography, ${ }^{41}$ and multiple ligation-dependent probe amplification. ${ }^{42}$

There are two limitations of the carrier test. First, $\sim 2 \%$ of cases with SMA arise as the result of de novo mutation events, 43 which is high when compared with most autosomal recessive disorders. The high rate of de novo mutations in SMN1 may account for the high carrier frequency in the general population despite the genetic lethality of the type I disease. The large number of repeated sequences around the SMN1 and SMN2 locus likely predisposes this region to unequal crossovers and recombination events and results in the high de novo mutation rate. The de novo mutations have been shown to occur primarily during paternal meiosis. ${ }^{43}$ Second, the copy number of SMN1 can vary on a chromosome; we have observed that $\sim 5 \%$ of the normal population possess three copies of SMN1.15 It is, therefore, possible for a carrier to possess one chromosome with two copies and a second chromosome with zero copies. ${ }^{2,15,44}$ Using haploid conversion technique, which allows for single chromosome analysis, Mailman et al. ${ }^{45}$ identified a parent of an affected child with a two-copy chromosome. The finding of two SMN1 genes on a single chromosome has serious genetic counseling implications because a carrier with two SMN1 genes on one

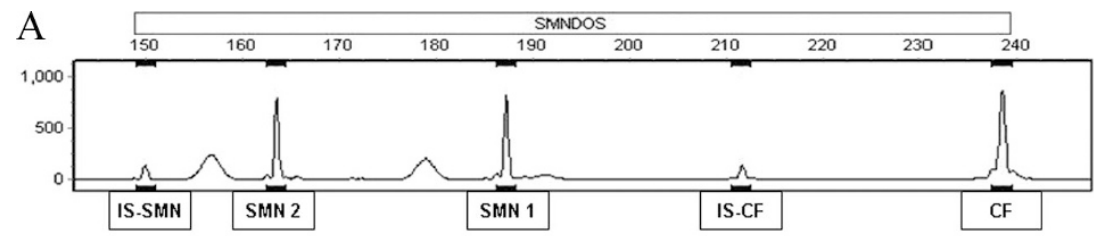

SMN1/SMN2

2,2 (noncarrier)

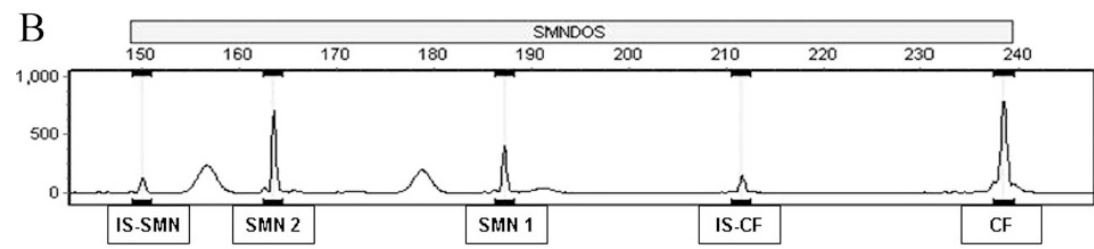

1,2 (Carrier)

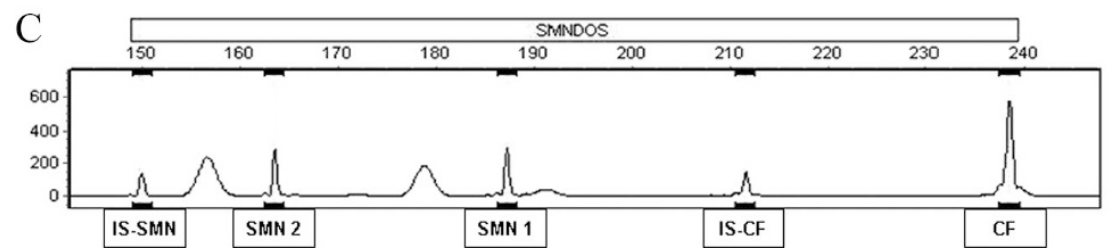

1,1 (Carrier)

Fig. 4. SMN1 dosage analysis of a normal control and two SMA carriers. The SMN1/CF ratio for the carrier is half the ratio of the normal control. The quantitative PCR assay uses an exon of the cystic fibrosis (CF) gene as a standard to determine the copy number of the SMN1 and SMN2 genes. The assay also incorporates the use of two internal standards (IS-CF and IS-SMN) to monitor the efficiency of the PCR reaction and to ensure that equal amounts of target genomic DNA are added to each tube. A, normal control with two SMN1 copies and two SMN2 copies. B, SMA carrier with one SMN1 copy and two SMN2 copies. C, SMA carrier with one SMN1 copy and one SMN2 copy. 
chromosome and a SMN1 deletion on the other chromosome will have the same dosage result as a noncarrier with one SMN1 gene on each Chromosome 5. Thus, the finding of normal two SMN1 copy dosage significantly reduces the risk of being a carrier; however, there is still a residual risk of being a carrier, and subsequently a small recurrence risk of future affected offspring for individuals with two SMN1 gene copies. Risk assessment calculations using Bayesian analysis are essential for the proper genetic counseling of SMA families. ${ }^{46} \mathrm{~A}$ recent report has shown that there are significant differences in carrier frequencies and the two-copy chromosome genotypes among different ethnic groups. ${ }^{47}$ The results from this study provide adjusted detection rates based on ethnicity, and thus allow for more accurate Bayesian risk estimates.

\section{Population carrier screening}

In current practice, patients with a family history of SMA are most often tested for carrier status. However, the American College of Medical Genetics recently recommended population carrier screening for SMA. ${ }^{2}$ Population carrier screening is currently recommended for a number of other genetic disorders with similar carrier frequencies. The prototype for heterozygote screening was testing for Tay-Sachs disease in the Ashkenazi Jewish population in whom carrier testing has been offered since 1969. Carrier screening, followed by prenatal diagnosis when indicated, has resulted in a dramatic decrease in the incidence of Tay-Sachs disease in the Jewish population. ${ }^{48}$ It is generally accepted that the following criteria should be met in order for a screening program to be successful: (1) disorder is clinically severe, (2) high frequency of carriers in the screened population, (3) availability of a reliable test with a high specificity and sensitivity, (4) availability of prenatal diagnosis, and (5) access to genetic counseling. SMA does meet the criteria cited. However, the choice to have a SMA carrier test should be made by an informed decision. Educational brochures are available and provide information about SMA and the inheritance patterns. ${ }^{49}$ It is important that individuals undergoing carrier testing recognize that the test does not provide genotype/phenotype information. Type I SMA occurs in $\sim 60 \%$ to $70 \%$ of the cases, whereas the milder types II and III account for the remaining cases. ${ }^{5}$ Formal genetic counseling services must be made available to anyone requesting this testing. It is important that all individuals undergoing testing understand that a carrier is a healthy individual who is not at risk of developing the disease but has a risk of passing the gene mutation to his/her offspring. Family planning options are available to parents and include: egg or sperm donation, adoption, preimplantation genetic testing, and termination of pregnancy. It is imperative that individuals understand the limitations of the molecular testing: two SMN1 genes in cis on the one Chromosome 5, presence or rare de novo mutations, and nondeletion mutations. As is true for all carrier screening programs, the testing must be voluntary.

\section{Protein}

SMA results from an insufficient amount of the SMN protein and there is a strong correlation between the disease severity and SMN protein levels. ${ }^{27,28}$ The SMN protein is a ubiquitously expressed, highly conserved 294-amino acid polypeptide. The protein is found in both the cytoplasm and nucleus and is concentrated in punctate structures call "gems" in the nucleus. ${ }^{50}$ High levels of the protein have been found to exist in the spinal motor neurons, the affected cells in patients with SMA. The protein self-associates into a multimeric structures. Biochemically, SMN does not appear to exist within cells in isolation but instead forms part of a large protein complex, the SMN com- plex. ${ }^{51}$ Many of these SMN-interacting proteins are components of various ribonucleoprotein (RNP) complexes that are involved in distinct aspects of RNA metabolism. The best characterized function of the SMN complex is regulating the assembly of a specific class of RNA-protein complexes, the small nuclear RNPs (snRNPs). ${ }^{52}$ The snRNPs are a critical component of the spliceosome; a large RNA-protein that catalyzes pre-mRNA splicing.

Because the SMN protein is ubiquitously expressed, it remains unknown how a loss of a general housekeeping function (snRNP assembly) causes a selective loss of motor neurons in SMA. ${ }^{53}$ The high expression of SMN protein in motor neurons may suggest that the neuronal population is more sensitive to decreases in the SMN protein level. Possibly, the altered splicing of a unique set of pre-mRNAs results in deficient proteins that are necessary for motor neuron growth and survival. However, specific alterations in mRNAs have not been identified in patients with SMA. In addition to its role in spliceosomal RNP assembly, SMN may have other functions in motor neurons. A subset of SMN complexes are located in axons and growth cones of motor neurons and may be involved in some aspects of axonal transport and localized translation of specific mRNAs. ${ }^{54,55}$

\section{Therapy}

Management depends on treating or preventing complications associated with weakness and maintaining the quality of life. Pulmonary disease is the major cause of morbidity and mortality in type I SMA. Pulmonary compromise is caused by a combination of inspiratory and expiratory muscle weakness. Respiratory muscle weakness results in impaired cough and ability to clear lower airway secretions, lung and chest wall underdevelopment, and hypoventilation. Respiratory care of patients with SMA is essential to their survival and quality of life. ${ }^{56}$ Chronic respiratory management includes providing methods for airway clearance and noninvasive ventilatory support. In SMA type I patients with increasingly frequent acute pulmonary infections, tracheotomy and ventilation can be considered but may not improve the quality of life or reduce hospitalizations. Discussion with the family about the management of respiratory failure should occur early in the disease and continue in an ongoing dialogue. Attention to nutritional status and to orthopedic complications, such as corrective spinal surgery for scoliosis, is also important.

Strategies are being actively investigated to increase the expression of full-length SMN protein levels from the SMN2 gene. Several compounds have now been shown to up-regulate SMN expression by inhibiting histone deacetylase (HDAC). Control of the acetylation state of histones is an important epigenetic mechanism regulating gene expression. When the $\mathrm{NH}-2$ terminus of core histones is acetylated in a region of chromatin, this region takes on a more relaxed chromatin structure that is more transcriptionally active due to increased accessibility of DNA to the transcriptional machinery. The level of histone acetylation is controlled by both the histone acetyltransferases, which acetylate the histones, and the HDACs, which deacetylate histones. Chang et al. ${ }^{57}$ demonstrated that sodium butyrate, one of the earliest discovered HDAC inhibitors, increased full-length SMN2 transcript levels and protein levels in lymphoblastoid cell lines derived from type I patients. Subsequent studies have shown that the SMN2 promoter can be activated and full-length SMN RNA and protein levels increased by several other HDAC inhibitors including phenylbutyrate and valproic acid (VPA). Both of these drugs have been in clinical use for many years for other indications and have 
well-established safety profiles and consequently are now being used in SMA clinical trials. ${ }^{58,59}$ A pilot study of phenylbutyrate showed that the drug was well tolerated. ${ }^{59}$ Two small open-label trials of VPA have reported modest strength or functional benefit in a subset of patients with SMA. ${ }^{60,61}$ A phase II openlabel study of VPA indicated that the drug could be used safely in patients with SMA older than 2 years, and there was some evidence in support of improvement in gross motor function in younger nonambulatory type II children. ${ }^{62}$ The authors concluded that the study underscores the need for additional controlled clinical trial with VPA. ${ }^{62}$

A drug that has been proposed to work by activating SMN2 expression is hydroxyurea (HU), another HDAC inhibitor in clinical use for many years. HU has previously shown to activate the fetal hemoglobin gene and has been used to treat patients with sickle cell disease and thalassemias. HU was shown to increase the amount of full-length transcript and protein in patient-derived lymphoblastoid cell lines and is currently being used in clinical trials in Taiwan and in the United States. ${ }^{63}$

Another possible treatment strategy would be to enhance SMN2 exon 7 inclusion by using synthetic antisense oligonucleotides that bind SMN2 transcripts and promote exon 7 inclusion during splicing. ${ }^{64,65}$ Although such strategies have been successful in vitro, it will be a greater challenge to achieve efficient delivery of the oligonucleotides to the motor neuron in patients with SMA.

\section{CONCLUSIONS}

As a result of the discovery of the $S M N$ gene and elucidation of the mutational spectrum, clinical diagnostic testing for SMA has significantly improved. It is no longer necessary to perform the invasive muscle biopsy in most cases. Until an effective treatment is found to cure or arrest the progression of the disease, prevention of new cases through accurate diagnosis and carrier and prenatal diagnosis is of the utmost importance. The goal of population-based SMA carrier screening is to identify couples at risk for having a child with SMA, thus allowing carriers to make informed reproductive choices. In the future, newborn screening for SMA may become a reality and allow for the implementation of more proactive treatments. The correlation between the SMA phenotype and the SMN2 copy number in patients with SMA and the demonstration that sufficient SMN protein from SMN2 in transgenic mice can ameliorate the disease has made the SMN2 gene an obvious target that can be modulated in therapeutic strategies. Several compounds have now been shown to up-regulate the SMN protein from the SMN2 gene and are being used in clinical trials.

\section{REFERENCES}

1. Pearn J. Incidence, prevalence, and gene frequency studies of chronic childhood spinal muscular atrophy. J Med Genet 1978;15:409-413.

2. Prior TW. Carrier screening for spinal muscular atrophy. Genet Med 2009; 10:20-26.

3. Munstat TL, Davies KE. International SMA consortium meeting. Neuromuscul Disord 1992;2:423-428.

4. Zerres K, Rudnik-Schoneborn S. Natural history in proximal spinal muscular atrophy: clinical analysis of 445 patients and suggestions for a modification of existing classifications. Arch Neurol 1995;52:518-523.

5. Meldrum C, Scott C, Swoboda KJ. Spinal muscular atrophy genetic counseling access and genetic knowledge: parents perspective. J Child Neurol 2007;22:1019-1026.

6. Feldkotter M, Schwarzer V, Wirth R, Wienker TF, Wirth B. Quantitative analysis of SMN1 and SMN2 based on real-time LightCycler PCR: fast and highly reliable carrier testing and prediction of severity of spinal muscular atrophy. Am J Hum Genet 2002;70:358-368.

7. Alias L, Bernal S, Fuentes-Prior P, et al. Mutation update of spinal muscular atrophy in Spain: molecular characterization of 745 unrelated patients and identification of four novel mutations in the SMNI gene. Hum Genet 2009; 125:29-39.

8. Brzustowicz LM, Lehner T, Castilla LH, et al. Genetic mapping of chronic childhood-onset spinal muscular atrophy to chromosome 5 q11.213.3. Nature $1990 ; 244: 540-541$

9. Melki J, Sheth P, Abdelhak S, et al. Mapping of acute (type I) spinal muscular atrophy to chromosome $5 \mathrm{q} 12-\mathrm{q} 14$. The French Spinal Muscular Atrophy Investigators. Lancet 1990;336:271-273.

10. Lefebvre S, Burglen L, Reboullet S, et al. Identification and characterization of a spinal muscular atrophy-determining gene. Cell 1995;80:155-165.

11. Bürglen L, Lefebvre S, Clermont $\mathrm{O}$, et al. Structure and organization of the human survival motor neuron (SMN) gene. Genomics 1996;32:479-482.

12. Monani UR, McPherson JD, Burghes AH. Promoter analysis of the human centromeric and telomeric survival motor neuron genes (SMNC and SMNT). Biochim Biophys Acta 2007;1445:330-336.

13. Echaniz-Laguna A, Miniou P, Bartholdi D, Melki J. The promoters of the survival motor neuron gene $(S M N)$ and its copy $(S M N c)$ share common regulatory elements. Am J Hum Genet 1999;64:1354-1370.

14. Campbell L, Potter A, Ignatius J, Dubowitz V, Davies K. Genomic variation and gene conversion in spinal muscular atrophy: implications for disease process and clinical phenotype. Am J Hum Genet 1997;61:40-50.

15. McAndrew PE, Parsons DW, Simard LR, et al. Identification of proximal spinal muscular atrophy carriers and patients by analysis of SMNT and SMNC gene copy number. Am J Hum Genet 1997;60:1411-1422.

16. Wirth B, Herz M, Wetter A, et al. Quantitative analysis of survival motor neuron copies: identification of subtle SMN1 mutations in patients with spinal muscular atrophy, genotype-phenotype correlation, and implications for genetic counseling. Am J Hum Genet 1999;64:1340-1356.

17. Mailman MD, Heinz JW, Papp AC, et al. Molecular analysis of spinal muscular atrophy and modification of the phenotype by SMN2. Genet Med 2002;4:20-26.

18. Prior TW, Swoboda KJ, Scott HD, Hejmanowski AQ. Homozygous SMN1 deletions in unaffected family members and modification of the phenotype by SMN2. Am J Med Genet A 2004;130:207-310.

19. Hsieh-Li HM, Chang JG, Jong YJ, et al. A mouse model for spinal muscular atrophy. Nat Genet 2000;26:66-70.

20. Monani UR, Coovert DD, Burghes AH. Animal models of spinal muscular atrophy. Hum Mol Genet 2000;9:2451-2457.

21. Hoffman Y, Lorson CL, Stamm S, Androphy EJ, Wirth B. Htra2-beta 1 stimulates an exonic splicing enhancer and can restore full-length SMN expression to survival motor neuron 2 (SMN2). Proc Natl Acad Sci USA 2000;97:9618-9623.

22. Oprea GE, Krober S, McWhorter ML, et al. Plastin 3 is a protective modifier of autosomal recessive spinal muscular atrophy. Science 2008;320:524-527.

23. Lorson CL, Androphy EJ. An exonic enhancer is required for inclusion of an essential exon in the SMA-determining gene SMN. Hum Mol Genet 2000; 9:259-265.

24. Lorson CL, Hahnen E, Androphy EJ, Wirth B. A single nucleotide in the $S M N$ gene regulates splicing and is responsible for spinal muscular atrophy. Proc Natl Acad Sci USA 1999;96:6307-6311.

25. Cartegni L, Kraniner AR. Disruption of an SF2/ASF-dependent exonic splicing enhancer in $S M N 2$ causes spinal muscular atrophy in the absence of SMN1. Nat Genet 2002;30:377-384.

26. Kashima T, Manley JL. A negative element in $S M N 2$ exon 7 inhibits splicing in spinal muscular atrophy. Nat Genet 2003;34:460-463.

27. Lefebvre S, Burlet P, Liu Q, et al. Correlation between severity and SMN protein level in spinal muscular atrophy. Nat Genet 1997;16:265-269.

28. Coovert DD, Le TT, McAndrew PE, et al. The survival motor neuron protein in spinal muscular atrophy. Hum Mol Genet 1997;6:1205-1214.

29. Gavrilov DK, Shi X, Das K, Gilliam TC, Wang CH. Differential SMN2 expression associated with SMA severity. Nat Genet 1998;20:230-231.

30. Prior TW, Krainer AR, Hua Y, et al. A positive modifier of spinal muscular atrophy in the SMN2 gene. Am J Hum Genet 2009;85:408-413.

31. van der Steege G, Grootscholten P, van der Vlies, et al. PCR-based DNA test to confirm clinical diagnosis of autosomal recessive spinal muscular atrophy. Lancet 1995;345:985-986.

32. Swoboda KJ, Prior TW, Scott CB, et al. Natural history of denervation in SMA: relation to age, SMN2 copy number, and function. Ann Neurol 2005;57:704-712.

33. Green NS, Pass KA. Neonatal screening by DNA microarray: spots and chips. Nat Rev Genet 2005;6:147-151.

34. Saxena A. Issues in newborn screening. Genet Test 2003;7:131-134

35. Pyatt RE, Mihal DC, Prior TW. Assessment of liquid microbead arrays for the screening of newborns for spinal muscular atrophy. Clin Chem 2007; 53:1879-1885

36. Lorson CL, Strasswimmer J, Yao JM, et al. SMN oligomerization defect correlates with spinal muscular atrophy severity. Nat Genet 1998;19:63-66.

37. Wirth B. An update of the mutation spectrum of the survival motor neuron gene $(S M N 1)$ in autosomal recessive spinal muscular atrophy. Hum Mutat 2000; $15: 228-237$. 
38. Parsons DW, McAndrew PE, Iannaccone ST, Mendell JR, Burghes AH, Prior TW. Intragenic telSMN mutations: frequency, distribution, evidence of a founder effect and modification of spinal muscular atrophy phenotype by cenSMN copy number. Am J Hum Genet 1998;63:1712-1723.

39. Gerard B, Ginet N, Matthijs G, et al. Genotype determination at the survival motor neuron locus in a normal population and SMA carriers using competitive PCR and primer extension. Hum Mutat 2000;16:253-263.

40. Anhuf D, Eggermann T, Rudnik-Schoneborn S, Zerres K. Determination of SMN1 and SMN2 copy number using TaqMan technology. Hum Mutat 2003;22:74-78.

41. Su Y-N, Hung C-C, Li H, et al. Quantitative analysis of SMN1 and SMN2 genes based on DHPLC: a highly efficient and reliable carrier-screening test. Hum Mutat 2005;25:460-467.

42. Huang $\mathrm{CH}$, Chang YY, Chen $\mathrm{CH}$, et al. Copy number analysis of survival motor neuron genes by multiplex ligation-dependent probe amplification. Genet Med 2007;9:241-248.

43. Wirth B, Schmidt T, Hahnen E. De novo rearrangements found in $2 \%$ of index patients with spinal muscular atrophy: mutational mechanisms, parental origin, mutation rate and implications for genetic counseling. Am J Hum Genet 1997;61:1102-1111.

44. Ogino S, Leonard DG, Rennert H, Ewens WJ, Wilson RB. Genetic risk assessment in carrier testing for spinal muscular atrophy. Am J Med Genet 2002;110:301-307.

45. Mailman MD, Hemingway T, Darsey RL, et al. Hybrids monosomal for human chromosome 5 reveal the presence of a spinal muscular atrophy (SMA) carrier with two SMN1 copies on one chromosome. Hum Genet 2001;108:109-115.

46. Ogino S, Wilson RB. Genetic testing and risk assessment for spinal muscular atrophy. Hum Genet 2002;111:477-500.

47. Hendrickson BC, Donohoe C, Akmaev VR, et al. Differences in SMN1 allele frequencies among ethnic groups within North America. $J$ Med Genet 2009;46:641-644.

48. Kaback MM. Screening and prevention in Tay-Sachs disease: origins, update, and impact. Adv Genet 2001;44:253-265.

49. Claire Altman Heine Foundation Inc., for the Prevention of spinal muscular atrophy. Available at: www.prevent sma.org. Accessed April 17, 2008.

50. Liu Q, Fischer U, Wang F, Drefuss G. The spinal muscular atrophy gene product, SMN, and its associated protein SIP1 are in a complex with spliceosomal snRNP proteins. Cell 1997;90:1013-1021.

51. Paushkin S, Gubitz AK, Massenet S, Dreyfuss G. The SMN complex, an assemblyosome of ribonucleoproteins. Curr Opin Cell Biol 2002;14:305312

52. Yong J, Wan L, Dreyfuss G. Why do cells need an assembly machine for RNA-protein complexes? Trends Cell Biol 2004;14:226-232.

53. Monani UR. Spinal muscular atrophy: a deficiency in a ubiquitous protein; a motor neuron-specific disease. Neuron 2005;48:885-896.

54. Zhang HL, Pan F, Hong D, Shenoy SM, Singer RH, Bassell GJ. Active transport of the survival motor neuron protein and the role of exon-7 in cytoplasmic localization. J Neurosci 2003;23:6627-6637.

55. Rossoll W, Jablonka S, Andreassi C, et al. Smn, the spinal muscular atrophy-determining gene product, modulates axon growth and localization of beta-actin mRNA in growth cones of motoneurons. $J$ Cell Biol 2003;163: $801-812$.

56. Iannaccone ST. Modern management of spinal muscular atrophy. $J$ Child Neurol 2007;22:974-978.

57. Chang JG, Hsieh-Li HM, Jong YJ, Wang NM, Tsai CH, Li H. Treatment of spinal muscular atrophy by sodium butyrate. Proc Nat Acad Sci USA 2001;98:9808-9813.

58. Brichita L, Holker I, Huang K, Klockgether T, Wirth B. In vivo activation of SMN in spinal muscular atrophy carriers and patients treated with valproate. Ann Neurol 2006;59:970-975.

59. Brahe C, Vitali T, Tiziano FD, et al. Phenylbutyrate increases SMN gene expression in spinal muscular atrophy patients. Eur J Hum Genet 2005;13: $356-359$.

60. Weihl CC, Connolly AM, Pestronk A. Valproate may improve strength and function in patients with type III/IV spinal muscular atrophy. Neurology 2006;67:500-501.

61. Tsai LK, Yang CC, Hwu WL, Li H. Valproic acid treatment in six patients with spinal muscular atrophy. Eur J Neurol 2007;14:e8-e9.

62. Swoboda KJ, Scott CB, Reyna SP. Phase II open label study of valproic acid in spinal muscular atrophy. PLoS One 2009;4:e5268.

63. Grzeschik SM, Ganta M, Prior TW, Heavlin WD, Chang CH. Hydroxyurea enhances SMN2 expression in spinal muscular atrophy. Ann Neurol 2005; 58:194-202.

64. Cartegni L, Krainer AR. Correction of disease-associated exon skipping by synthetic exon-specific activators. Nat Struct Biol 2003;10:120-125.

65. Skordis LA, Dunckley MG, Yue B, Eperon IC, Muntoni F. Bifunctional antisense oligonucleotides provide a trans-acting splicing enhancer that stimulates SMN2 expression in patient fibroblasts. Proc Natl Acad Sci USA 2003;100:4114-4119. 